\title{
Microscale Marangoni Surfers
}

\author{
Kilian Dietrich $\odot,{ }^{1}$ Nick Jaensson $\odot,{ }^{2, *}$ Ivo Buttinoni, ${ }^{3}$ Giorgio Volpe $\odot,{ }^{4}$ and Lucio Isa $\odot^{1, \dagger}$ \\ ${ }^{1}$ Laboratory for Soft Materials and Interfaces, Department of Materials, ETH Zürich, 8093 Zürich, Switzerland \\ ${ }^{2}$ Laboratory for Soft Materials, Department of Materials, ETH Zürich, 8093 Zürich, Switzerland \\ ${ }^{3}$ Institut für Experimentelle Kolloidphysik, Heinrich-Heine Universität Düsseldorf, D-40225 Düsseldorf, Germany \\ ${ }^{4}$ Department of Chemistry, University College London, 20 Gordon Street, London WC1H OAJ, United Kingdom
}

(Received 14 May 2020; accepted 23 July 2020; published 25 August 2020)

\begin{abstract}
We apply laser light to induce the asymmetric heating of Janus colloids adsorbed at water-oil interfaces and realize active micrometric "Marangoni surfers." The coupling of temperature and surfactant concentration gradients generates Marangoni stresses leading to self-propulsion. Particle velocities span 4 orders of magnitude, from microns/s to $\mathrm{cm} / \mathrm{s}$, depending on laser power and surfactant concentration. Experiments are rationalized by finite elements simulations, defining different propulsion regimes relative to the magnitude of the thermal and solutal Marangoni stress components.
\end{abstract}

DOI: 10.1103/PhysRevLett.125.098001

Microscale active materials constituted by ensembles of self-propelling colloidal particles offer tremendous opportunities for fundamental studies on systems far from equilibrium and for the development of disruptive technologies [1]. Central to their functions is the ability to convert uniformly distributed sources of energy, i.e., under the form of chemical fuel or external driving fields, into net motion thanks to built-in asymmetry in their geometry or composition. Both from a modeling and a control perspective, minimalistic designs are particularly appealing. In such designs, the complexity of the particles is kept to a minimum, while still enabling functionality and the emergence of novel physical behaviors. The simplest case is the one of active Janus microspheres, i.e., colloidal beads equipped with a surface patch of a different material, which exploit their broken symmetry to self-propel and yet reveal a broad range of complex phenomena, including dynamic clustering [2,3], swarming [4], and guided motion [5].

Self-motility in Janus particles can derive from various mechanisms-from catalytic reactions [6] to bubble propulsion [7] and electrokinetic effects [8]. Among the available propulsion schemes, self-phoretic mechanisms have emerged as a standard [9]. In self-phoresis, a particle propels with a velocity that is proportional to a selfgenerated gradient via a phoretic mobility coefficient [10]. Self-thermophoresis, whereby motion is induced by the asymmetric heating of light-absorbing Janus particles [11], is particularly interesting due to the unique properties of light as a source of self-propulsion [12]. Here, the propulsion velocity is $\boldsymbol{V}=-D_{T} \nabla T$, where both the thermophoretic mobility $D_{T}$ [13] and the self-generated thermal gradient are independent of particle size [14]. The propulsion speed simply scales with incident illumination in a linear fashion, enabling robust possibilities for spatial and temporal motion control by light modulation [15-17].
However, direct self-thermophoresis in bulk liquids is not an efficient propulsion mechanism. Its molecular origin stems from particle-solvent interactions and the magnitude of $D_{T}$ is set by the temperature dependence of the particle(solid)-liquid interfacial energy [18]. Because the latter quantity depends weakly on temperature, thermophoretic mobilities are small. Typical values of $D_{T}$ are $\mathcal{O}\left(10^{-12} \mathrm{~m}^{2} / \mathrm{s}\right)$, leading to speeds of $\mathcal{O}(\mu \mathrm{m} / \mathrm{s})$, i.e., just a few body lengths per second for micrometric colloids, for an increase of the cap temperature $(\Delta T)$ of $1 \mathrm{~K}$, as confirmed by experiments $[11,14,17]$ and theoretical predictions [19]. A powerful way to improve the efficiency of thermal gradients for self-propulsion relies on coupling them to other gradients, such as asymmetric chemical gradients $[20,21]$.

In this Letter, by employing Janus particles at fluid-fluid interfaces, we couple thermal gradients to gradients of interfacial tension. Upon heating, controlled surface tension differences across the particle can lead to selfpropulsion velocities up to staggering $10^{4}$ body lengths per second, a vast increase over direct self-thermophoresis in bulk. This enhancement follows the fact that, in the presence of surface tension gradients, momentum conservation at the interface prescribes the existence of tangential stresses, called Marangoni stresses, defined as

$$
\nabla_{s} \sigma(\Gamma, T)=\frac{\partial \sigma(\Gamma, T)}{\partial T} \nabla_{s} T+\frac{\partial \sigma(\Gamma, T)}{\partial \Gamma} \nabla_{s} \Gamma,
$$

where $\sigma(\Gamma, T)$ is the interfacial tension, which is a function of temperature $T$ and surface excess concentration of surface-active species $\Gamma . \nabla_{s}=(\boldsymbol{I}-\boldsymbol{n n}) \cdot \nabla$ is the surface gradient operator, with $\boldsymbol{I}$ the unit tensor and $\boldsymbol{n}$ the normal to the interface. Here, we identify two sources of stress: temperature and surface excess concentration gradients, 
whose magnitude is set by $\partial \sigma(\Gamma, T) / \partial T$ and $\partial \sigma(\Gamma, T) / \partial \Gamma$, respectively [22]. Imposing a force balance on the particle's surface $\partial P$ and contact line $L$ yields

$$
\int_{\partial P} \boldsymbol{\sigma} \cdot \boldsymbol{n}_{p} d S=\int_{L} \sigma(\Gamma, T) \boldsymbol{t} d l
$$

where $\boldsymbol{\sigma}$ is the bulk stress tensor, $\boldsymbol{n}_{p}$ is the unit vector normal to the particle surface, and $t$ is the unit vector tangential to the interface and normal to the contact line. Together with a no-slip boundary condition at the particle surface, Eq. (2) allows solving for the particle velocity $\boldsymbol{V}$ imposed by the Marangoni stress [23]. For a characteristic interfacial tension difference $\Delta \sigma$, simple dimensional arguments lead to predicting a propulsion speed $V \propto \Delta \sigma / \eta$, where $\eta$ is an effective viscosity experienced by the particle straddling the interface. Considering thermal Marangoni effects (a.k.a. thermocapillarity) alone, the predicted selfpropulsion speed is given by $V \approx(\partial \sigma / \partial T) \Delta T /(10 \eta)$ [24]. Typical values of $\partial \sigma / \partial T$ for oil-water interfaces are $\mathcal{O}\left(10^{-4} \mathrm{~N} /(\mathrm{m} \cdot \mathrm{K})\right)$, leading to speeds $V=\mathcal{O}(\mathrm{cm} / \mathrm{s})$, independent of particle size and indeed corresponding to $10^{4}$ body lengths per second for microparticles, for $\Delta T=$ $1 \mathrm{~K}$ and $\eta=\mathcal{O}\left(10^{-3} \mathrm{~Pa} \cdot \mathrm{s}\right)$ [24]. The magnitude of $\partial \sigma / \partial T$ is then able to set macroscopic bodies in motion, as for instance shown for the propulsion of centimeter-sized objects [37] and the rotation of microgears suspended at a water-air interface [38]. Similar considerations can be made for solutal Marangoni propulsion [39,40], as popularized by camphor or soap "boats" releasing surfactant at one end [41,42], or for the motion of active droplets [43].

Our interfacial microswimmers, or "Marangoni surfers," are Janus silica microparticles (radius $R=3.15 \mu \mathrm{m}$, Microparticles $\mathrm{GmbH}$ ) sputter-coated with a $100 \mathrm{~nm}$ thick hemisphere of gold (CCU-010, safematic) and confined at an interface between MilliQ water and dodecane (Arcos Organics, purified three times through a basic alumina column). By pinning the lower aqueous phase to the sharp edges of a metal ring before adding a layer of dodecane, we achieve a macroscopically flat interface (area $\approx 0.8 \mathrm{~cm}^{2}$ ) to which the particles are added via contacting the interface with a $0.5 \mu \mathrm{l}$ droplet of a diluted aqueous suspension $(0.01 \% \mathrm{w} / \mathrm{v})$. The surface heterogeneity generated by the thick metallic caps effectively pins the Janus particles in random orientations with respect to the interface [44,45], leading to caps typically crossing the interface (Fig. S1 in [23]). Asymmetric heating of the particles is achieved by illuminating them with green laser light $(2 \mathrm{~W}-\mathrm{CW}$, Coherent Verdi, $532 \mathrm{~nm}$ ). In particular, we use beam-shaping optics to transform a Gaussian laser profile into a top-hat profile [Fig. 1(a)] focused onto the interface plane to provide localized, spatially uniform illumination (within 10\% intensity fluctuations) with a power density up to $8000 \mathrm{~W} / \mathrm{cm}^{2}$ [23]. Light absorption by the gold cap creates an asymmetric temperature profile around the particles [23], thus generating

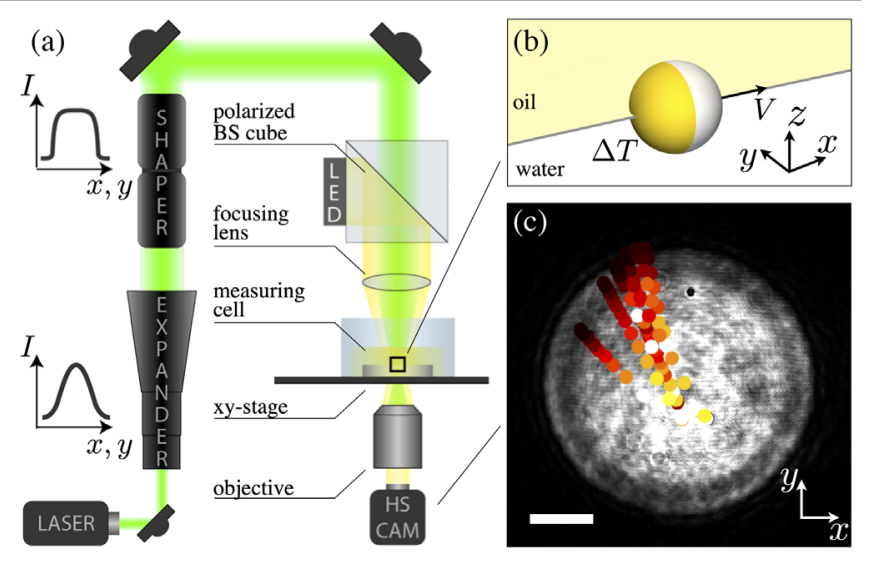

FIG. 1. (a) Diagram of the experimental setup. (b) Schematic of a gold-coated Janus particle at the water-oil interface. Laser illumination induces a temperature increase $\Delta T$ in the fluid in contact with the cap, leading to asymmetric Marangoni stresses and particle propulsion with velocity $V$. (c) Overlay of different trajectories of Marangoni surfers propelling from the center of the illuminated region. The color code indicates normalized velocity ranging between zero (black) and a power-density-dependent $V_{\max }$ (white). Scale bar $=30 \mu \mathrm{m}$.

Marangoni stresses that propel them with velocity $V$ and the Au cap oriented toward the back [Fig. 1(b)] [19]. Trajectories are collected by positioning a particle in the center of the illuminated circular spot, turning the laser on at a given power density and recording images with a high-speed camera (AX 200 mini, Photron, up to $5000 \mathrm{fps}$ ) in a custom-built transmission microscope [Fig. 1(c)]. From the high-speed time lapses, we extract particle coordinates and velocities via custom-written MATLAB particle-tracking algorithms. Particles self-propel from the center toward the periphery of the light spot in random directions, depending on the cap orientation (Movie S1) and are recentered in the laser spot after each measurement. As soon as the particles leave the laser spot, propulsion stops (Movie S2). Light gradients are only present at the periphery of the spot ( $\sim$ outer $15 \%$ of the spot radius), and we observe no coupling to propulsion direction. Particle speed, as a function of laser illumination, reaches values up to $1 \mathrm{~cm} / \mathrm{s}$. Variations of particle speed for a given illumination may stem from differences in particle orientation and position (i.e., threephase contact angle) relative to the interface and from differences in the metal coating.

In order to rationalize the phenomenology seen in the experiments, we perform systematic numerical simulations of the fluid dynamics of the system coupled to heat and mass transport of surfactants at the interface using an in-house finite element code (full details in [23]). In particular, as an ansatz to quantify the Marangoni stresses introduced in Eq. (1), we assume that, in first approximation, the surface tension can be described by a linear function of $\Gamma$ and $T$ [25]:

$$
\sigma(\Gamma, T)=\sigma_{0}-\delta \Gamma-\beta\left(T-T_{0}\right),
$$


where $\sigma_{0}$ is the surface tension of the clean interface at the ambient temperature $T_{0} . \delta$ and $\beta$ are the previously introduced parameters describing how the surface tension changes with $\Gamma$ and $T$, respectively identified with $-\partial \sigma / \partial \Gamma$ and $-\partial \sigma / \partial T$. Simulations were performed for Janus microparticles at a water-dodecane interface, where the model parameters were either known or taken from the literature [23]. The results include the temperature, surface excess concentration, and velocity fields as a function of $\Delta T$, which is the difference between the temperature of fluids in contact with the cap and $T_{0}$ and the equilibrium surfactant concentration $\Gamma_{0}$.

Starting from the case of a pristine interface $\left(\Gamma_{0}=0\right)$, in Fig. 2 we plot the simulated particle velocities $V$ as a function of $\Delta T$ (purple data). Here, we see that the speed increases roughly linearly with $\Delta T$. A dimensional analysis reveals that for fixed material parameters and in the absence of surfactants, the problem can be described by a single dimensionless group, for which we choose the thermal Péclet number, defined by $P e_{T}=2 V R /\left(\alpha_{1}+\alpha_{2}\right)$, where $\alpha_{i}$ are the thermal diffusivities of each liquid. Isosurfaces of the dimensionless temperature fields $T^{*}=\left(T-T_{0}\right) / \Delta T$ for the clean interface are shown in Fig. 2 for $\Delta T=1 \mathrm{~K}$ (top, left) and $20 \mathrm{~K}$ (top, right), respectively corresponding to the case of $P e_{T} \ll 1$ and $P e_{T} \simeq 1$. For $P e_{T} \ll 1$, the problem is governed by thermal diffusion, and the temperature fields can be described as a combination of a monopole and a dipole solution. As the cap heating increases, the particle speed, and thus $P e_{T}$, increase, too, until convection starts affecting the temperature field, resulting in a stretched region of increased $T$ behind the particle. A direct comparison with existing theoretical analysis by Würger [24] shows very good agreement for the particle $V$ even in the high $P e_{T}$ regime [23].

When we include the effect of surface-active species at the interface (green, blue, and orange data), we obtain a markedly different behavior that strongly depends on the value of $\Gamma_{0}$. We reveal the existence of a regime showing a linear velocity increase for low values of $\Delta T$, which expands over to broader ranges of $\Delta T$ for increasing surfactant surface excess, i.e., from green to orange data. However, the corresponding velocities are several orders of magnitude lower than the ones for the clean interface, even for a surface excess concentration as low as $10^{-7} \mathrm{~mole} / \mathrm{m}^{2}$. Interestingly, for this value of $\Gamma_{0}$ (green data), as $\Delta T$ grows, the particle speed goes through a transition regime and it converges toward the values for the clean interface. By increasing $\Gamma_{0}$, this transition takes place at a correspondingly higher $\Delta T$.

In order to understand this behavior, we visualize the surface excess concentration fields around the particles and revert once more to dimensional analysis. As compared to the clean interface, we need to introduce two additional dimensionless groups: a surface Péclet number, defined $P e_{s}=V R / D_{s}$, where $D_{s}$ is the surface diffusion

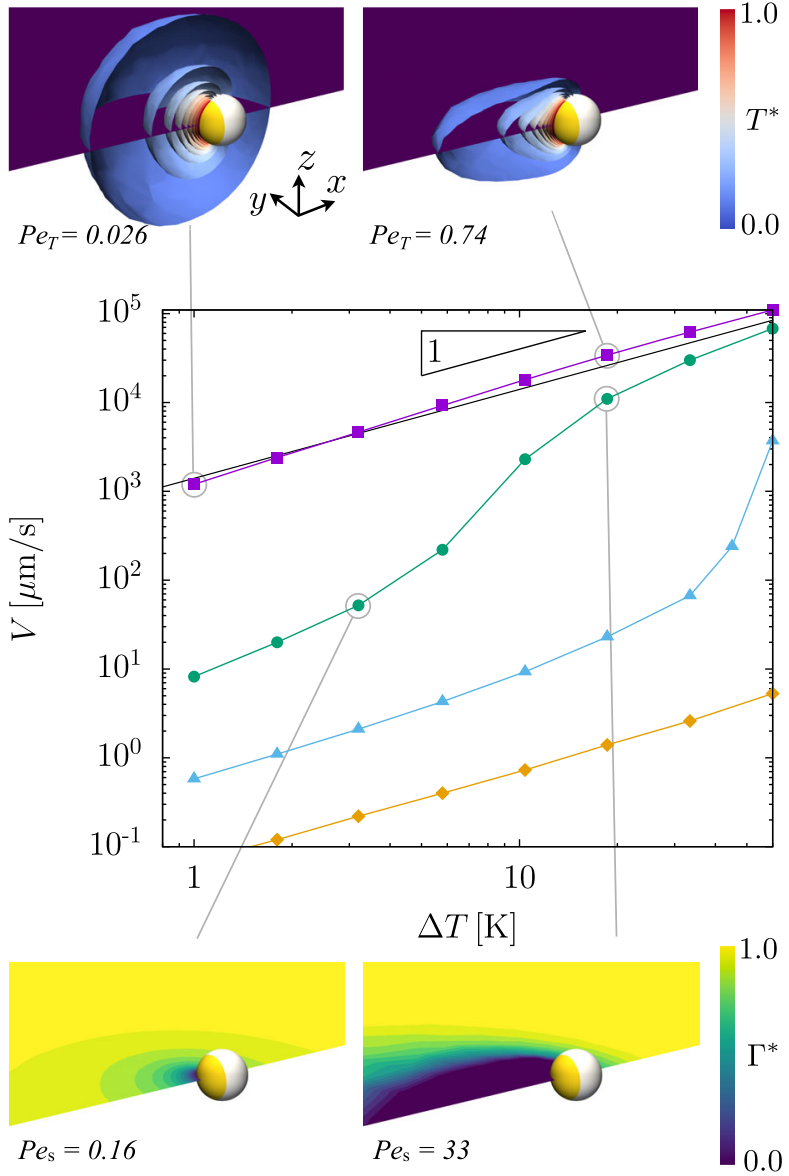

FIG. 2. Simulated particle speed versus temperature increase $\Delta T$ in the fluid next to the cap of a Janus particle at a pristine water-dodecane interface (purple squares) and with excess concentrations $\Gamma_{0}=10^{-7}$ (green circles), $10^{-6}$ (blue triangles), and $10^{-5} \mathrm{~mole} / \mathrm{m}^{2}$ (orange diamonds). The symbols are simulation results, and the connecting lines are to guide the eye. Solid black line: theoretical prediction by Würger [24]. The insets show isosurfaces of the dimensionless temperature $T^{*}=\left(T-T_{0}\right) / \Delta T$ (top) and excess concentration $\Gamma^{*}=\Gamma / \Gamma_{0}$ (bottom) fields for selected simulations corresponding to different regimes of thermal and surface Péclet numbers $P e_{T}$ and $P e_{s}$, respectively.

coefficient, and the ratio between solutal and thermal Marangoni stresses, $\Pi=\beta \Delta T /\left(\delta \Gamma_{0}\right)$ [46]. Since the surface diffusion coefficient can easily be 2 orders of magnitude lower than the thermal diffusion coefficient [47], the transition $P e_{s} \simeq 1$ happens at a correspondingly lower $\Delta T$, for which the surface excess concentration field starts to deviate significantly from the diffusion-dominated regime. The dimensionless surface excess concentration fields shown in Fig. 2 (bottom) reveal that, as the particle moves, a wake depleted of surfactants is created behind it. Therefore, the corresponding surface concentration gradient generates solutal Marangoni stresses that "work against" the thermal Marangoni stresses. The resulting particle velocity is caused by the balance between the 
two stress components as in Eq. (1), which explains the slowdown. The consequent transition to velocities purely dominated by thermal stresses happens because, at high $P e_{s}$, the surface excess concentration inside the depleted region becomes approximately zero. At this point, the maximum attainable solutal Marangoni stress, fixed by $\delta \Gamma_{0}$, is reached and becomes independent of temperature, or particle speed, afterward. Conversely, the maximum thermal Marangoni stress is set by $\beta \Delta T$, which keeps growing as the temperature increases. At a given point, thermal Marangoni stresses dominate, and the presence of the surfactant becomes insignificant, so that the data in Fig. 2 for a clean interface sets an upper limit for the particle velocity at a given $\Delta T$.

Starting from these numerical predictions, we closely examine the measured experimental particle speeds as a function of incident laser power, which we convert into a $\Delta T$, leading to the data reported in Fig. 3. We perform the conversion by carefully calibrating the local heating of the fluids induced by the gold caps relative to the critical temperature of a water-lutidine mixture. We show a linear relation between the induced heating and incident laser power, as supported by theoretical estimates (see Supplemental Material [23]). We first perform a series of measurements at an allegedly pristine water-dodecane interface. The purple data show a behavior consistent with the scenario reported by the simulations, where, in spite of all efforts for cleanliness, solutal effects are always present. For comparison, the simulation results are also plotted (empty symbols) and the experimental behavior can be

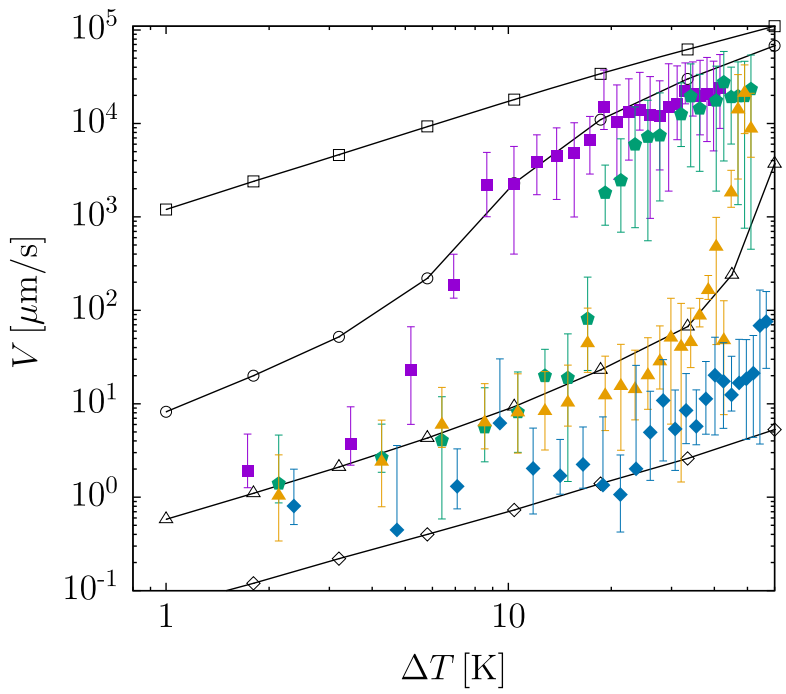

FIG. 3. Experimental particle speed $V$ as a function of $\Delta T$. Allegedly pristine interface (purple squares) and increasing concentrations of SDS in the water phase: $C=10^{-7}$ (green pentagons), $10^{-5}$ (orange triangles), and $10^{-3} \mathrm{~mole} / \mathrm{L}$ (blue diamonds). Black, open symbols are simulated data with the symbol shape corresponding to Fig. 2. The error bars represent the standard deviations of particle velocities. reconciled by introducing a surface excess concentration of order $\Gamma_{0}=10^{-7} \mathrm{~mole} / \mathrm{m}^{2}$. These minute values of $\Gamma_{0}$ correspond to unavoidable environmental trace contaminations [48-50], which have a hardly measurable effect on the absolute level of the surface tension. However, as low as the absolute levels are, gradients of the surface excess concentration can still significantly alter the hydrodynamics in sensitive experiments, especially at small length scales, where Marangoni stresses become increasingly important [51].

To confirm the role played by surface-active species, we purposely add controlled amounts of a water-soluble surfactant (sodium dodecyl sulfate, SDS, Sigma Aldritch, $\geq 98.5 \%$ ). The choice of SDS is motivated by the fact that it has a negligible surface viscosity, and thus we do not expect surface rheology to affect particle motion [52]. Consistent with the balance between the different components of Marangoni stresses, we observe that an increased amount of SDS causes a shift of the transition toward higher values of $\Delta T$ and an overall reduced particle velocity over the same $\Delta T$ window. To unify the experimental and numerical data, and to unequivocally show that the ratio between the solutal and thermal Marangoni stresses controls the transition between the two regimes, we can rescale the experimental and simulation data on a master curve as a function of the dimensionless number $\Pi$ in Fig. 4. Below $\Pi=1$, the data clearly collapses onto a single curve, with a transition that happens at $\Pi=1$ for all the data shown. We remark that the data only collapses for $\Pi<1$; for larger values of $\Pi$ the role of the solutal Marangoni stresses becomes insignificant and $V$ is rescaled by $\Delta T$ alone.

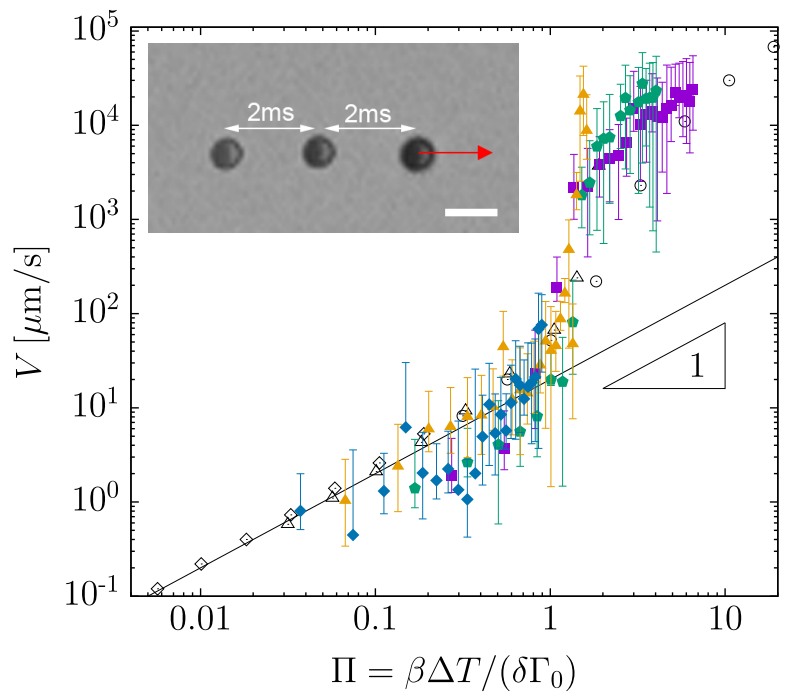

FIG. 4. Rescaled experimental and simulation data. For the experiments the excess concentrations used are $\Gamma_{0}=2 \times 10^{-7}$, $4 \times 10^{-7}, 1 \times 10^{-6}$, and $2 \times 10^{-6} \mathrm{~mole} / \mathrm{m}^{2}$ for SDS concentrations of $C=0,10^{-7}, 10^{-5}$, and $10^{-3} \mathrm{~mole} / \mathrm{L}$, respectively. The symbols and error bars correspond to the ones in Fig. 3. The solid black line is a guide to the eye. Inset: Image overlay of a particle propelling with $V \simeq 10 \mathrm{~mm} / \mathrm{s}$ (scale bar $=10 \mu \mathrm{m})$. 
The understanding and rationalization of the experimental data opens up exciting opportunities for the exploitation of Marangoni stresses in self-propelled, active microscale systems. The green and purple data in Figs. 3 and 4 show that the propulsion velocity of a microscale Marangoni surfer can be tuned over 4 orders of magnitude in a single experiment via the controlled balance between thermal and solutal effects. In particular, the fact that this huge dynamic range can be regulated simply by light enables unprecedented opportunities for the spatial and temporal modulation of self-propulsion. The existence of two distinct linear regimes at low and high $\Pi$ allows fine-tuning of propulsion speed within two markedly different velocity ranges. At low $\Delta T$, velocities are on the order of $1-10 \mu \mathrm{m} / \mathrm{s}$, as typical for active Brownian particles. The simultaneous illumination of multiple particles with controlled light landscapes offers interesting options to modulate collective active motion. In the high $\Delta T$ regime, the particles move fully ballistically with speeds reaching up to $20 \mathrm{~mm} / \mathrm{s}$ (inset of Fig. 4), which had so far only been reported for bubble propulsion of microscale objects [53]. Moreover, the narrow transition region together with the steep velocity variation can give rise to rich dynamical behavior crossing between regions of low and high $P e \propto V / \sqrt{D_{T} D_{R}}$ for active motion, where $D_{T}$ and $D_{R}$ are the Brownian translational and rotational diffusivities. Finally, the strong dependence of propulsion speed on interface contamination may be used as a sensitive characterization tool for the presence of surfaceactive species undetectable by macroscopic tensiometry methods.

In conclusion, from the demonstration of the first catalytically active particle onward [54], fluid interfaces have been offering a broad range of promising opportunities to realize new active systems [55], exploiting the unique combination of strong vertical confinement [56,57], specific interactions [58], and highly efficient available propulsion sources. We expect that the near future will see further expansion encompassing both fundamental studies and applications [59].

The authors thank M. A. Hulsen at the Eindhoven University of Technology (TU/e) for access to the TFEM software libraries, and A. Studart, M. Fiebig, and E. Dufresne at ETH Zürich and A. Fink at the Adolphe Merkle Institute for access to instrumentation. J. Vermant at ETH Zürich and R. Piazza at Politecnico di Milano are acknowledged for insightful comments. N. J. acknowledges TOTAL S. A. for financial support. L. I. and K.D. acknowledge financial support from the ETH Research Grant No. ETH-16 15-1. L. I. and G. V. acknowledge financial support from the MCSA-ITN-ETN "ActiveMatter" Grant agreement No. 812780. G. V. acknowledges sponsorship for this work by the U.S. Office of Naval Research Global (Award No. N6290918-1-2170).
K. D. and N.J. contributed equally to this work. Author contributions are defined based on the CRediT (Contributor Roles Taxonomy) and listed alphabetically. Conceptualization: I. B., K. D., L. I. Formal analysis: K. D., L. I., N. J., G. V. Funding acquisition: I. B., L. I. Investigation: K. D., N. J., G. V. Methodology: I. B., K. D., L. I., N. J., G. V. Project administration: L. I. Supervision: I. B, L. I. Validation: K. D., N. J. Visualization: K. D., L. I., N. J. Writing-original draft: K. D., L. I., N. J. Writing-review and editing: I. B., K. D., L. I., N. J., G. V.

*Corresponding author. n.jaensson@gmail.com Corresponding author. lucio.isa@mat.ethz.ch

[1] C. Bechinger, R. Di Leonardo, H. Löwen, C. Reichhardt, G. Volpe, and G. Volpe, Rev. Mod. Phys. 88, 045006 (2016).

[2] I. Buttinoni, J. Bialké, F. Kümmel, H. Löwen, C. Bechinger, and T. Speck, Phys. Rev. Lett. 110, 238301 (2013).

[3] J. Palacci, S. Sacanna, A. P. Steinberg, D. J. Pine, and P. M. Chaikin, Science 339, 936 (2013).

[4] J. Yan, M. Han, J. Zhang, C. Xu, E. Luijten, and S. Granick, Nat. Mater. 15, 1095 (2016).

[5] J. Simmchen, J. Katuri, W. E. Uspal, M. N. Popescu, M. Tasinkevych, and S. Sánchez, Nat. Commun. 7, 10598 (2016).

[6] S. Sanchez, L. Soler, and J. Katuri, Angew. Chem., Int. Ed. Engl. 54, 1414 (2015).

[7] R. Wang, W. Guo, X. Li, Z. Liu, H. Liu, and S. Ding, RSC Adv. 7, 42462 (2017).

[8] S. Gangwal, O. J. Cayre, M. Z. Bazant, and O. D. Velev, Phys. Rev. Lett. 100, 058302 (2008).

[9] J. Moran and J. Posner, Annu. Rev. Fluid Mech. 49, 511 (2017).

[10] J. L. Anderson, Annu. Rev. Fluid Mech. 21, 61 (1989).

[11] H.-R. Jiang, N. Yoshinaga, and M. Sano, Phys. Rev. Lett. 105, 268302 (2010).

[12] P. Zemánek, G. Volpe, A. Jonáš, and O. Brzobohatý, Adv. Opt. Photonics 11, 577 (2019).

[13] M. Braibanti, D. Vigolo, and R. Piazza, Phys. Rev. Lett. 100, 108303 (2008).

[14] A. P. Bregulla and F. Cichos, Faraday Discuss. 184, 381 (2015).

[15] O. Ilic, I. Kaminer, Y. Lahini, H. Buljan, and M. Soljačić, ACS Photonics 3, 197 (2016).

[16] B. Qian, D. Montiel, A. Bregulla, F. Cichos, and H. Yang, Chem. Sci. 4, 1420 (2013).

[17] A. P. Bregulla, H. Yang, and F. Cichos, ACS Nano 8, 6542 (2014).

[18] A. Parola and R. Piazza, Eur. Phys. J. E 15, 255 (2004).

[19] T. Bickel, A. Majee, and A. Würger, Phys. Rev. E 88, 012301 (2013).

[20] I. Buttinoni, G. Volpe, F. Kümmel, G. Volpe, and C. Bechinger, J. Phys. Condens. Matter 24, 284129 (2012).

[21] J. R. Gomez-Solano, S. Samin, C. Lozano, P. RuedasBatuecas, R. van Roij, and C. Bechinger, Sci. Rep. 7, 14891 (2017). 
[22] V.-M. Ha and C.-L. Lai, Proc. Math. Phys. Eng. Sci. 457, 885 (2001).

[23] See Supplemental Material at http://link.aps.org/ supplemental/10.1103/PhysRevLett.125.098001 for full experimental and numerical details, which also includes Refs. [19,20,24-36].

[24] A. Würger, J. Fluid Mech. 752, 589 (2014).

[25] G. Homsy and E. Meiburg, J. Fluid Mech. 139, 443 (1984).

[26] H.-R. Jiang, N. Yoshinaga, and M. Sano, Phys. Rev. Lett. 105, 268302 (2010).

[27] R. L. Olmon, B. Slovick, T. W. Johnson, D. Shelton, S.-H. Oh, G. D. Boreman, and M. B. Raschke, Phys. Rev. B 86, 235147 (2012).

[28] H. Stone, Phys. Fluids A 2, 111 (1990).

[29] C. Geuzaine and J.-F. Remacle, Int. J. Numer. Methods Eng. 79, 1309 (2009).

[30] A. Brooks and T. Hughes, Comput. Methods Appl. Mech. Eng. 32, 199 (1982).

[31] R. Glowinski, T. Hesla, and D. Joseph, Int. J. Multiphase Flow 25, 755 (1999).

[32] N. Jaensson, M. Hulsen, and P. Anderson, J. NonNewtonian Fluid Mech. 235, 125 (2016).

[33] N. Jaensson, M. Hulsen, and P. Anderson, Comput. Fluids 156, 81 (2017).

[34] M. Villone, G. D'Avino, M. Hulsen, F. Greco, and P. Maffettone, Comput. Fluids 42, 82 (2011).

[35] CRC Handbook of Chemistry and Physics, 100th ed., edited by J. Rumble (CRC Press, Boca Raton, 2019).

[36] S. Zeppieri, J. Rodríguez, and A. López De Ramos, J. Chem. Eng. Data 46, 1086 (2001).

[37] D. Okawa, S. Pastine, A. Zettl, and Fréchet, J. Am. Chem. Soc. 131, 5396 (2009).

[38] C. Maggi, F. Saglimbeni, M. Dipalo, F. De Angelis, and R. Di Leonardo, Nat. Commun. 6, 7855 (2015).

[39] E. Lauga and A. Davis, J. Fluid Mech. 705, 120 (2012).

[40] H. Masoud and H. Stone, J. Fluid Mech. 741, 1 (2014).

[41] R. J. Strutt, Proc. R. Soc. London 47, 364 (1890).
[42] S. Sur, H. Masoud, and J. P. Rothstein, Phys. Fluids 31, 102101 (2019).

[43] C. Maass, C. Krüger, S. Herminghaus, and C. Bahr, Annu. Rev. Condens. Matter Phys. 7, 171 (2016).

[44] D. J. Adams, S. Adams, J. Melrose, and A. C. Weaver, Colloids Surf. A 317, 360 (2008).

[45] X. Wang, M. In, C. Blanc, P. Malgaretti, M. Nobili, and A. Stocco, Faraday Discuss. 191, 305 (2016).

[46] T. Bickel, Eur. Phys. J. E 42, 131 (2019).

[47] D. Wang, L. Pevzner, C. Li, K. Peneva, C. Y. Li, D. Y. C. Chan, K. Müllen, M. Mezger, K. Koynov, and H.-J. Butt, Phys. Rev. E 87, 012403 (2013).

[48] B. Liu, R. Manica, Q. Liu, E. Klaseboer, Z. Xu, and G. Xie, Phys. Rev. Lett. 122, 194501 (2019).

[49] A. Maali, R. Boisgard, H. Chraibi, Z. Zhang, H. Kellay, and A. Würger, Phys. Rev. Lett. 118, 084501 (2017).

[50] O. Manor, I. U. Vakarelski, X. Tang, S. J. O’Shea, G. W. Stevens, F. Grieser, R. R. Dagastine, and D. Y. C. Chan, Phys. Rev. Lett. 101, 024501 (2008).

[51] C. Brennen, Cavitation and Bubble Dynamics (Cambridge University Press, Cambridge, England, 2014).

[52] Z. A. Zell, A. Nowbahar, V. Mansard, L. G. Leal, S. S. Deshmukh, J. M. Mecca, C. J. Tucker, and T. M. Squires, Proc. Natl. Acad. Sci. U.S.A. 111, 3677 (2014).

[53] S. Sanchez, A. N. Ananth, V. M. Fomin, M. Viehrig, and O. G. Schmidt, J. Am. Chem. Soc. 133, 14860 (2011).

[54] R. Ismagilov, A. Schwartz, N. Bowden, and G. Whitesides, Angew. Chem., Int. Ed. Engl. 41, 652 (2002).

[55] W. Fei, Y. Gu, and K. Bishop, Curr. Opin. Colloid Interface Sci. 32, 57 (2017).

[56] K. Dietrich, D. Renggli, M. Zanini, G. Volpe, I. Buttinoni, and L. Isa, New J. Phys. 19, 065008 (2017).

[57] X. Wang, M. In, C. Blanc, M. Nobili, and A. Stocco, Soft Matter 11, 7376 (2015).

[58] K. Dietrich, G. Volpe, M. N. Sulaiman, D. Renggli, I. Buttinoni, and L. Isa, Phys. Rev. Lett. 120, 268004 (2018).

[59] T. Yao, N. G. Chisholm, E. B. Steager, and K. J. Stebe, Appl. Phys. Lett. 116, 043702 (2020). 